\title{
Yeast Fermentation Product Improves Pre-transit Antioxidant Defense and Post-transit Performance of Beef Steers
}

DOI:10.31274/air.12244

Erin L. Deters, Post-Doctoral Research Associate; Stephanie L. Hansen, Professor;

Department of Animal Science, Iowa State University

Summary and Implications

Steers supplemented Diamond V NaturSafe, a yeast fermentation product, at the manufacturer's current recommended dose for receiving cattle $(12 \mathrm{~g} / \mathrm{steer} / \mathrm{d})$ during a 19-d preconditioning period exhibited greater antioxidant (glutathione) capacity prior to a 19-h transit event. Regardless of treatment, activity of the antioxidant enzyme Mn-superoxide dismutase was increased posttransit, suggesting more antioxidants may be needed to combat transit-induced stress. Supplementing NaturSafe at $12 \mathrm{~g} /$ steer/d during both preconditioning and receiving also improved feedlot performance early in the receiving period ( $\mathrm{d} 0$ to 30 ). These data suggest increasing antioxidant status may be an effective strategy to help cattle prepare for and recover from a stressful event, such as long-distance transit.

\section{Introduction}

Beef cattle experience various stressors during the feedlot receiving period including weaning, vaccination, commingling, and transportation. This often results in increased incidence of disease and poor performance upon arrival at the feedlot. Preconditioning programs have been shown to improve the subsequent health and performance of feedlot cattle. NaturSafe is a yeast (Saccharomyces cerevisiae) fermentation product designed to promote rumen health and balanced immunity in beef cattle. Therefore, NaturSafe may be a beneficial addition to preconditioning and/or receiving cattle diets. The objectives of this study were 1) to determine the effect of varying doses of NaturSafe on receiving period performance and antioxidant defense; 2) to determine the effects of supplementing NaturSafe during preconditioning (prior to a long-distance transit event) on subsequent receiving period performance; and 3) to examine changes in antioxidant enzyme activity relative to transit.

\begin{abstract}
Materials and Methods
This study utilized 48 newly weaned crossbred beef steers $(567 \pm 40 \mathrm{lb})$ from a single-source (IA). The trial was divided into two phases separated by a 19-h transit event: preconditioning (d -19 to -1 [start of transit event]) and receiving ( $\mathrm{d} 0$ [end of transit event] to 58). Seven days after arrival at the Iowa State Beef Nutrition Farm (d -19) steers were processed, weighed, and stratified by BW into preconditioning pens $(\mathrm{n}=1$ pen/treatment; 12 steers/pen) and pens randomly assigned to dietary treatments: NaturSafe (NS; Diamond V, Cedar Rapids, IA) at 0 (control; CON), 12 (NS12), or 18 (NS18) $\mathrm{g} /$ steer/d during both preconditioning and receiving or 0 $\mathrm{g} /$ steer/d during preconditioning then $18 \mathrm{~g} /$ steer/d during receiving (CON18). Treatments were delivered as part of the diet (Table 1) using dried distillers grains as a carrier. Back calculated NS intakes for each treatment are reported in Table 2 (preconditioning) and Table 3 (receiving).

On d -1, steers were weighed, loaded onto a semitruck and transported $1,086 \mathrm{mi}$ over $19 \mathrm{~h}$ to simulate the stress associated with feedlot receiving. Upon return to ISU (d 0), steers were weighed, stratified by BW, and sorted into receiving pens (6 steers/pen; 12 steers/treatment; 2 pens/treatment) with GrowSafe bunks (GrowSafe Systems Ltd., Airdrie, Alberta, Canada). During the receiving period (d 0 to 58 ), CON, NS12, and NS18 steers remained on the same dietary treatments as preconditioning while CON18 steers were switched from $0 \mathrm{~g} \mathrm{NS} /$ steer/d during preconditioning to $18 \mathrm{~g} \mathrm{NS} /$ steer/d during receiving. Weights were collected prior to feeding on d $0,29,30,57$, and 58. Feed efficiency (G:F) was calculated from d 0 to 30,30 to 58 , and 0 to 58 from steer DMI and weight gain. On d 1, steers were implanted with Component E-S (Elanco Animal Health, Greenfield, IN). One steer from NS12 died during the course of the study from illness unrelated to treatment; data for this steer was removed from the entire study.

Liver biopsies were performed on all steers at the start (d -20) and end of preconditioning (d -3); liver samples were analyzed for total, oxidized, and reduced glutathione concentrations using a commercially available kit (\#703002, Cayman Chemical, Ann Arbor, MI). Blood was collected from all steers immediately prior to transit (d -1) as well as 1 and $8 \mathrm{~d}$ post-transit for analysis of red blood cell lysate total, manganese (Mn), and copper/zinc
\end{abstract}

Copyright $(02021$ by the Authors. This is an open access article published under the CC BY-NC license (https://creativecommons.org/licenses/by-nc/4.0/), which allows for non-commercial reuse with proper attribution. 
$(\mathrm{Cu} / \mathrm{Zn})$-superoxide dismutase (SOD) activity using a commercially available kit (\#706002, Cayman Chemical).

Data were analyzed as a completely randomized design using ProcMixed of SAS 9.4 (SAS Institute Inc., Cary, NC). Steer served as the experimental unit $(n=12$ steers/treatment) for receiving period variables. The model included the fixed effect of treatment. Contrast statements were constructed to determine the effects of NS dose (linear and quadratic) and timing (NS18 vs. CON18). Glutathione concentrations from d -20 were used as a covariate in analysis of $\mathrm{d}-3$ concentrations. Red blood cell lysate SOD activity was analyzed as repeated measures with the repeated effect of day. Significance was declared at $P \leq 0.05$ and tendencies from $0.05<P \leq$ 0.10 .

\section{Results and Discussion}

Common management practices implemented during preconditioning programs include castration, dehorning, respiratory vaccinations, and weaning prior to shipping. These practices are thought to be vital to the health and performance of feeder cattle. The preconditioning period in the current study served to mimic the group feeding style of preconditioning periods common on farms. Preconditioning period performance means are reported in Table 2. Regardless of treatment, the 19-h transit event resulted in approximately $7 \% \mathrm{BW}$ shrink. At the end of preconditioning (d -3), there was a quadratic effect of NS where, among treatments tested, NS12 had the greatest concentrations of total, oxidized, and reduced glutathione (Table 4; $P=0.06$ ). Greater concentrations of reduced glutathione, the form in which glutathione can function as an antioxidant, suggests these steers had greater antioxidant capacity prior to the transit event which could be beneficial as others have observed a decrease in antioxidant capacity in response to transit. Activity of the antioxidant enzyme Mn-SOD was increased post-transit, resulting in a greater $\mathrm{Mn}$ :total-SOD activity ratio (Figure 1; day $P \leq 0.01)$. Stress hormones have been shown to increase Mn-SOD, indicating a greater need for antioxidants during periods of stress.

There was a quadratic effect of NS on ADG and G:F from $\mathrm{d} 0$ to 30 , where among treatments NS12 exhibited the greatest performance (Table 3; $P \leq 0.04$ ). Improved performance early in the receiving period for NS12-fed steers suggests these cattle were better equipped to handle the stress of transit as well as a novel diet and environment. Greater antioxidant capacity prior to transit for NS12 may have contributed to improved performance by these steers if dietary nutrients were spared for growth rather than producing antioxidants to combat transitinduced stress. No effect of NS was observed in the second half of the receiving period, suggesting the greatest performance response to NS supplementation may be immediately after stress. Although there were no effects of supplementation timing (CON18 vs. NS18) in the current study, further investigation of timing (preconditioning vs. feedlot receiving) is warranted. Additionally, future research should focus on NS supplementation rates of $12 \mathrm{~g} / \mathrm{steer} / \mathrm{d}$ or less.

\section{Acknowledgements}

The authors wish to acknowledge the graduate and undergraduate research assistants of the Hansen Ruminant Nutrition Lab at Iowa State University as well as the Iowa State Beef Nutrition Farm crew. Additional thanks to Diamond $\mathrm{V}$ for financial support of this project. 
Table 1. Ingredient composition of diets fed to steers throughout the trial

\begin{tabular}{|c|c|c|}
\hline & $\begin{array}{l}\text { Preconditioning } \\
\text { (d -19 to }-1)\end{array}$ & $\begin{array}{l}\text { Receiving } \\
\text { (d } 0 \text { to } 58)\end{array}$ \\
\hline \multicolumn{2}{|l|}{ Ingredient, \% DM basis } & 61 \\
\hline Corn silage & 50 & 40 \\
\hline Dry-rolled corn & 20 & 30 \\
\hline Dried distillers grains ${ }^{1}$ & 28.15 & 28.15 \\
\hline Limestone & 1.4 & 1.4 \\
\hline Salt & 0.31 & 0.31 \\
\hline Rumensin ${ }^{2}$ & 0.0135 & 0.0135 \\
\hline Vitamin A and E premix ${ }^{3}$ & 0.1 & 0.1 \\
\hline Trace mineral premix ${ }^{4}$ & 0.024 & 0.024 \\
\hline \multicolumn{3}{|l|}{ Analyzed composition ${ }^{5}$} \\
\hline Crude protein & 13.2 & 13.7 \\
\hline NDF & 27.9 & 24.5 \\
\hline Ether extract & 5.1 & 5.1 \\
\hline
\end{tabular}

${ }^{1}$ Carrier for micro-ingredients and NaturSafe $\mathbb{R}$ (Diamond V, Cedar Rapids, IA) treatments

${ }^{2}$ Provided approximately $180 \mathrm{mg}$ monensin/steer/d (Elanco Animal Health, Greenfield, IN)

${ }^{3}$ Premix provided 2,200 IU vitamin A and $25 \mathrm{IU}$ vitamin E/ $\mathrm{kg}$ diet DM

${ }^{4}$ Provided trace minerals as recommended by the National Research Council

${ }^{5}$ Based on analysis from Dairyland, Inc. (Arcadia, WI)

Table 2. Summary of newly received beef steer performance during a 19-d preconditioning period $(\mathrm{n}=1$ pen/treatment; 12 steers/pen)

\begin{tabular}{|c|c|c|c|c|c|}
\hline \multirow[b]{2}{*}{ Trait } & \multicolumn{4}{|c|}{ Treatment $^{1}$} & \multirow[b]{2}{*}{ SD } \\
\hline & $\mathrm{CON}$ & NS12 & NS18 & CON18 & \\
\hline NS intake ${ }^{2}$ & 0.0 & 12.0 & 18.9 & 0.0 & - \\
\hline Initial BW, lb & 567 & 566 & 565 & 567 & 39 \\
\hline Final BW, lb & 638 & 630 & 626 & 639 & 40 \\
\hline DMI, lb/d & 13.3 & 13.3 & 13.4 & 14.1 & 0.39 \\
\hline $\mathrm{ADG}, \mathrm{lb} / \mathrm{d}$ & 3.76 & 3.38 & 3.17 & 3.78 & 0.781 \\
\hline G:F & 0.282 & 0.255 & 0.236 & 0.268 & 0.020 \\
\hline BW Shrink ${ }^{3}, \%$ & 6.9 & 7.1 & 7.1 & 7.1 & 0.1 \\
\hline
\end{tabular}

${ }^{1}$ Diamond V (Cedar Rapids, IA) NaturSafe (NS) at 0 (CON), 12 (NS12), or 18 (NS18) g/steer/d throughout preconditioning ( $\mathrm{d}-19$ to -1 ) and receiving ( 0 to 58 ), or $0 \mathrm{~g} /$ steer/d during preconditioning then $18 \mathrm{~g} /$ steer/d during receiving (CON18)

${ }^{2}$ Back calculated NS intake; g/steer/d

${ }^{3}$ Percent BW shrink measured after a 19-h (1,086 mi) transit event 
Table 3. Effect of dose and timing of NaturSafe supplementation on feedlot performance of beef steers during a 58-d receiving period.

\begin{tabular}{|c|c|c|c|c|c|c|c|c|}
\hline & \multicolumn{4}{|c|}{ Treatment $^{1}$} & \multirow[b]{2}{*}{ SEM $^{3}$} & \multicolumn{3}{|c|}{ Contrast $P$-value ${ }^{2}$} \\
\hline & $\begin{array}{c}\mathrm{CON} \\
\mathrm{n}=12 \\
\text { steers }\end{array}$ & $\begin{array}{l}\mathrm{NS} 12 \\
\mathrm{n}=11 \\
\text { steers }\end{array}$ & $\begin{array}{c}\mathrm{NS} 18 \\
\mathrm{n}=12 \\
\text { steers }\end{array}$ & $\begin{array}{c}\text { CON18 } \\
\mathrm{n}=12 \\
\text { steers }\end{array}$ & & Linear & Quadratic & Timing \\
\hline NS intake ${ }^{4}$ & 0.0 & 11.4 & 18.1 & 18.9 & - & - & - & - \\
\hline Initial BW, lb & 638 & 633 & 626 & 639 & 12.5 & 0.49 & 0.85 & 0.42 \\
\hline Final BW, lb & 842 & 852 & 828 & 832 & 15.2 & 0.58 & 0.32 & 0.83 \\
\hline \multicolumn{9}{|l|}{ DMI, lb/d } \\
\hline d 0 to 30 & 14.7 & 15.4 & 15.1 & 15.3 & 0.54 & 0.55 & 0.51 & 0.79 \\
\hline d 30 to 58 & 18.4 & 18.3 & 17.6 & 18.4 & 0.61 & 0.41 & 0.63 & 0.38 \\
\hline d 0 to 58 & 16.6 & 16.8 & 16.4 & 16.8 & 0.54 & 0.87 & 0.54 & 0.53 \\
\hline \multicolumn{9}{|l|}{$\mathrm{ADG}, \mathrm{lb} / \mathrm{d}$} \\
\hline $\mathrm{d} 0$ to 30 & 3.02 & 3.63 & 3.00 & 2.90 & 0.218 & 0.77 & 0.02 & 0.74 \\
\hline d 30 to 58 & 4.06 & 3.91 & 4.00 & 3.77 & 0.240 & 0.81 & 0.72 & 0.49 \\
\hline $\mathrm{d} 0$ to 58 & 3.52 & 3.77 & 3.48 & 3.32 & 0.187 & 0.98 & 0.24 & 0.53 \\
\hline \multicolumn{9}{|l|}{$\mathrm{G}: \mathrm{F}$} \\
\hline d 0 to 30 & 0.206 & 0.237 & 0.198 & 0.189 & 0.014 & 0.90 & 0.04 & 0.65 \\
\hline d 30 to 58 & 0.222 & 0.218 & 0.224 & 0.205 & 0.012 & 0.94 & 0.94 & 0.25 \\
\hline d 0 to 58 & 0.213 & 0.226 & 0.211 & 0.197 & 0.009 & 0.97 & 0.22 & 0.25 \\
\hline
\end{tabular}

${ }^{1}$ Diamond V (Cedar Rapids, IA) NaturSafe (NS) at 0 (CON), 12 (NS12), or18 (NS18) g/steer/d throughout preconditioning (d -19 to -1 ) and receiving (d 0 to 58 ), or $0 \mathrm{~g} /$ steer/d during preconditioning then $18 \mathrm{~g} /$ steer/d during receiving (CON18) ${ }^{2}$ Linear and quadratic contrast statements compare CON, NS12, and NS18; Timing contrast statement compares NS18 vs. CON18

${ }^{3}$ Highest SEM of any treatment reported

${ }^{4}$ Back calculated NS intake; g/steer/d 
Table 4. Effect of dose and timing of NaturSafe supplementation on liver glutathione concentrations of beef steers

\begin{tabular}{|c|c|c|c|c|c|c|c|c|}
\hline & \multicolumn{4}{|c|}{ Treatment $^{1}$} & \multirow[b]{2}{*}{ SEM $^{3}$} & \multicolumn{3}{|c|}{ Contrast $P$-value ${ }^{2}$} \\
\hline & $\mathrm{CON}$ & SCFP12 & SCFP18 & CON18 & & Linear & Quadratic & Timing \\
\hline \multicolumn{9}{|c|}{$\begin{array}{l}\text { Liver glutathione }^{4}, \mu \mathrm{M} \\
\mathrm{d}-20^{5}\end{array}$} \\
\hline Total & 1.54 & 1.91 & 1.69 & 1.86 & - & - & - & - \\
\hline Oxidized & 0.17 & 0.22 & 0.19 & 0.21 & - & - & - & - \\
\hline Reduced & 1.37 & 1.67 & 1.52 & 1.65 & - & - & - & - \\
\hline Ratio $^{6}$ & 0.114 & 0.130 & 0.122 & 0.124 & - & - & - & - \\
\hline \multicolumn{9}{|l|}{$d-3$} \\
\hline Total & 2.14 & 2.26 & 1.96 & 1.97 & 0.103 & 0.27 & 0.06 & 0.92 \\
\hline Oxidized & 0.27 & 0.28 & 0.23 & 0.24 & 0.017 & 0.11 & 0.06 & 0.54 \\
\hline Reduced & 1.87 & 1.99 & 1.73 & 1.73 & 0.090 & 0.34 & 0.06 & 0.94 \\
\hline Ratio & 0.143 & 0.139 & 0.131 & 0.138 & 0.006 & 0.07 & 0.65 & 0.29 \\
\hline \multicolumn{9}{|l|}{ d 59} \\
\hline Total & 2.14 & 1.93 & 2.02 & 2.08 & 0.101 & 0.29 & 0.31 & 0.63 \\
\hline Oxidized & 0.25 & 0.22 & 0.23 & 0.24 & 0.015 & 0.35 & 0.31 & 0.76 \\
\hline Reduced & 1.89 & 1.72 & 1.78 & 1.84 & 0.088 & 0.28 & 0.34 & 0.59 \\
\hline Ratio & 0.132 & 0.128 & 0.131 & 0.129 & 0.004 & 0.77 & 0.55 & 0.80 \\
\hline
\end{tabular}

${ }^{1}$ Diamond V (Cedar Rapids, IA) NaturSafe (NS) at 0 (CON), 12 (NS12), or18 (NS18) g/steer/d throughout preconditioning (d -19 to -1 ) and receiving (d 0 to 58), or $0 \mathrm{~g} /$ steer/d during preconditioning then $18 \mathrm{~g} /$ steer/d during receiving (CON18)

${ }^{2}$ Linear and quadratic contrast statements compare CON, NS12, and NS18; Timing contrast statement compares NS18 vs. CON18

${ }^{3}$ Highest SEM of any treatment reported

${ }^{4}$ Gluathione concentrations reported as $\mu \mathrm{M}$ per gram of wet tissue

${ }^{5}$ Values from d -20 (prior to treatment initiation) utilized as a covariate in analysis

${ }^{6}$ Ratio calculated by dividing oxidized by reduced glutathione concentrations 


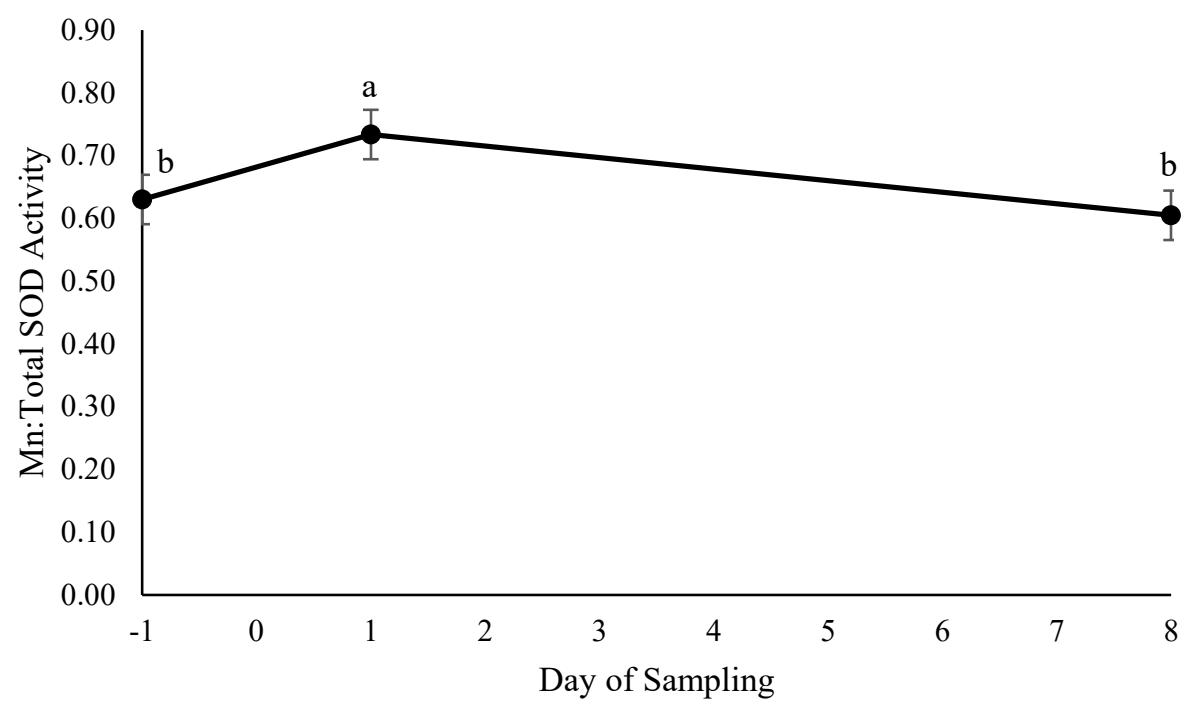

Figure 1. Effect of day $(P<0.01)$ on red blood cell lysate ratio of $\mathrm{Mn}$ to total-superoxide dismutase (SOD) activity in relation to a $19-\mathrm{h}(1,086 \mathrm{mi})$ transit event; steers were loaded on $\mathrm{d}-1$ and unloaded on $\mathrm{d} 0$. Treatment $\times$ day $P=0.71$. Unlike superscripts indicate a difference $(P<0.05)$ across days. 